\title{
Immunohistochemical Localization of Basement Membrane Proteins in Rat Ovarian Follicles
}

\author{
S. Casella, D.J. Schryver, Jr., T. Scott, J. Pflugheber, and K.J. Bodensteiner \\ Department of Biology, St. Lawrence University, 23 Romoda Drive, Canton, NY 13617
}

To survive, oocytes must associate with cells from the surrounding ovarian tissue. These cellular connections are established during follicular formation and are maintained throughout follicular development and oocyte maturation. Maintenance of follicular integrity is achieved, at least in part, via secretion of a basement membrane (BM) containing collagen IV, laminin, and fibronectin [1]. The BM is involved in cellular proliferation and differentiation and is essential for normal follicular development.

Large numbers of preantral follicles are necessary for the advancement of in vitro fertilization, embryo transfer, and cryopreservation technologies. Enzymatic dissociation of ovarian tissue is often used to obtain large numbers of preantral follicles for use in in vitro culture. However, use of enzymes in the dissociation process may damage or destroy the follicular BM. This, in turn, may lead to loss of normal follicular morphology in vitro. Therefore, knowledge of BM protein expression during normal follicular growth is essential for the development of a system able to sustain these cells in vitro.

Confocal microscopy and immunocytochemical staining were used to visualize the components of the BM in rat ovarian follicles throughout the early stages of folliculogenesis. Ovaries from adult rats were fixed in $4 \%$ paraformaldehyde and sections were cut at $8 \mu \mathrm{m}$. Slides were deparafinized and run through a graded series of alcohol. Slides labeled with Rabbit anti-laminin were permeabilized with $0.25 \%$ Triton-X for 20 min. Slides labeled with Rabbit anti-collagen IV were unmasked by boiling in a microwave for $10 \mathrm{~min}$ in a solution of Tris EDTA ( $\mathrm{pH} \mathrm{9.0).} \mathrm{Slides} \mathrm{labeled}$ with Mouse anti-fibronectin were unmasked in a solution of trisodium citrate ( $\mathrm{pH}$ 6.0). All slides were blocked with 10\% normal goat serum for one hour at room temperature, washed, and incubated with primary antibody for at least 8 hours $(5 \mu \mathrm{g} / \mathrm{ml}$ Rabbit anti-collagen, $1 \mu \mathrm{g} / \mathrm{ml} \mathrm{Rabbit} \mathrm{anti-laminin,}$ or $1 \mu \mathrm{g} / \mathrm{ml}$ Mouse anti-fibronectin). Slides were washed and incubated with the corresponding secondary antibody for three hours $(5 \mu \mathrm{g} / \mathrm{ml}$ Goat anti-mouse Alexa 546 or Goat anti-rabbit Alexa 546). Slides were then washed, mounted with Fluoromount-G, and visualized using confocal microscopy. Fibronectin, collagen IV, and laminin were present in the BM of ovarian follicles from primordial to early tertiary stages of follicular development.

These techniques can now be used to monitor the maintenance and/or repair of the BM in enzymatically isolated follicles cultured in vitro. Development of a culture system that allows for completion of follicular growth and oocyte maturation in vitro would have profound implications for the in vitro fertilization and embryo transfer industries [2].

[1] R.F. Rodgers et al., J Reprod Fertil Suppl. 54 (1999) 343.

[2] This work was supported by a St. Lawrence University Small Grant Award and the St. Lawrence University Fellows program. 

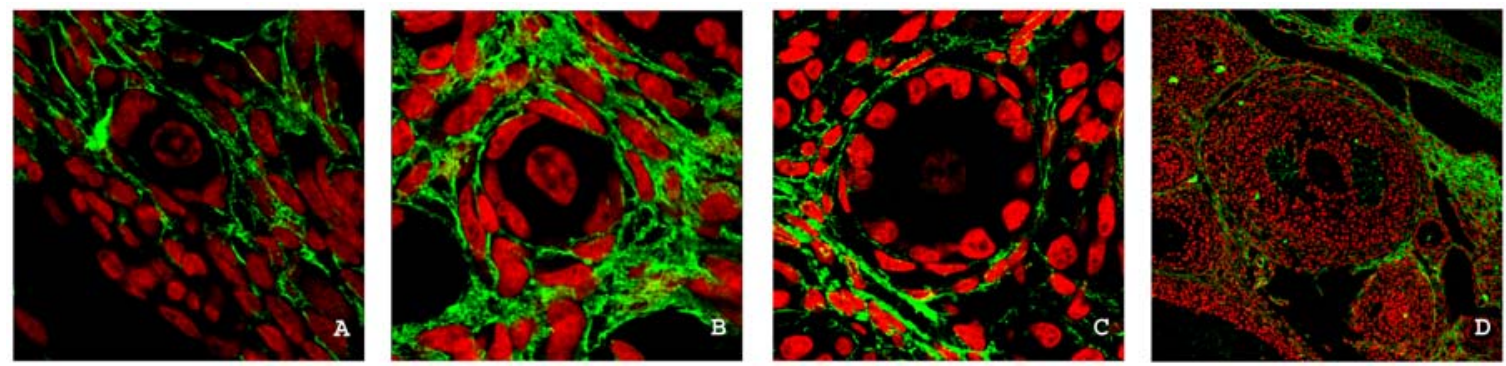

FIG 1: Immunohistochemical localization of fibronectin in the basement membrane of ovarian follicles from adult rats. All sections were stained with Mouse anti-Fibronectin (Green) and Draq-5 (Red). A and B) Primordial follicles. C) Primary follicle. D) Tertiary follicle. Images were taken at a magnification of 2199x, 2520x, 630x, and 200x, respectively.
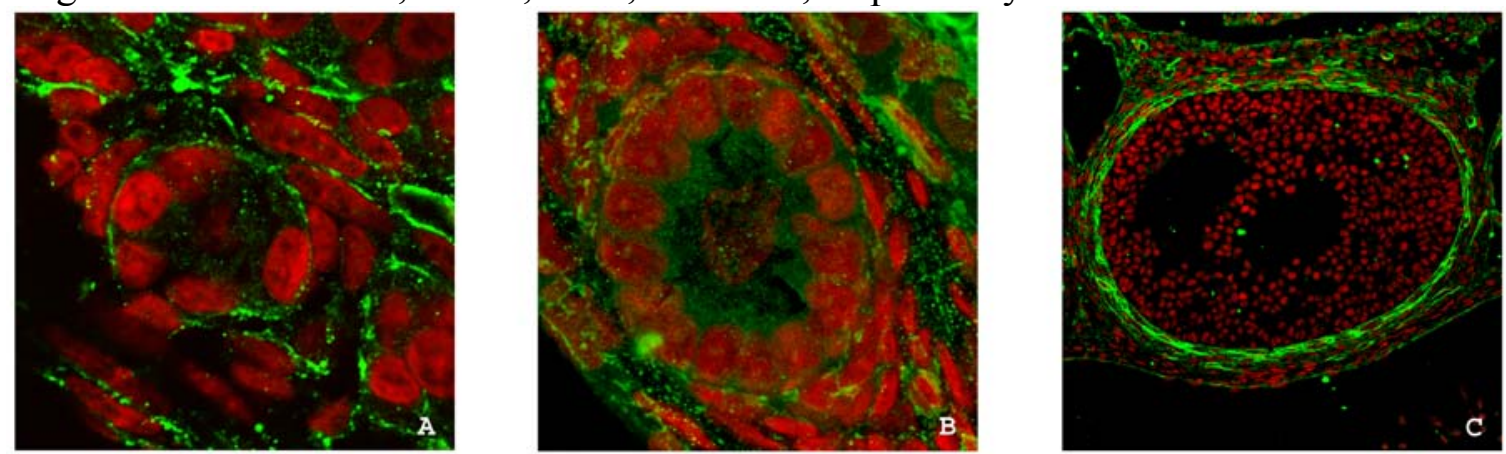

FIG 2: Immunohistochemical localization of collagen IV in the basement membrane of ovarian follicles from adult rats. All sections were stained with Rabbit anti-Collagen (Green) and Draq-5 (Red). A) Primordial follicle. B) Primary follicle. C) Tertiary follicle. Images were taken at a magnification of $2867 x, 2520 x$, and $384 x$, respectively.
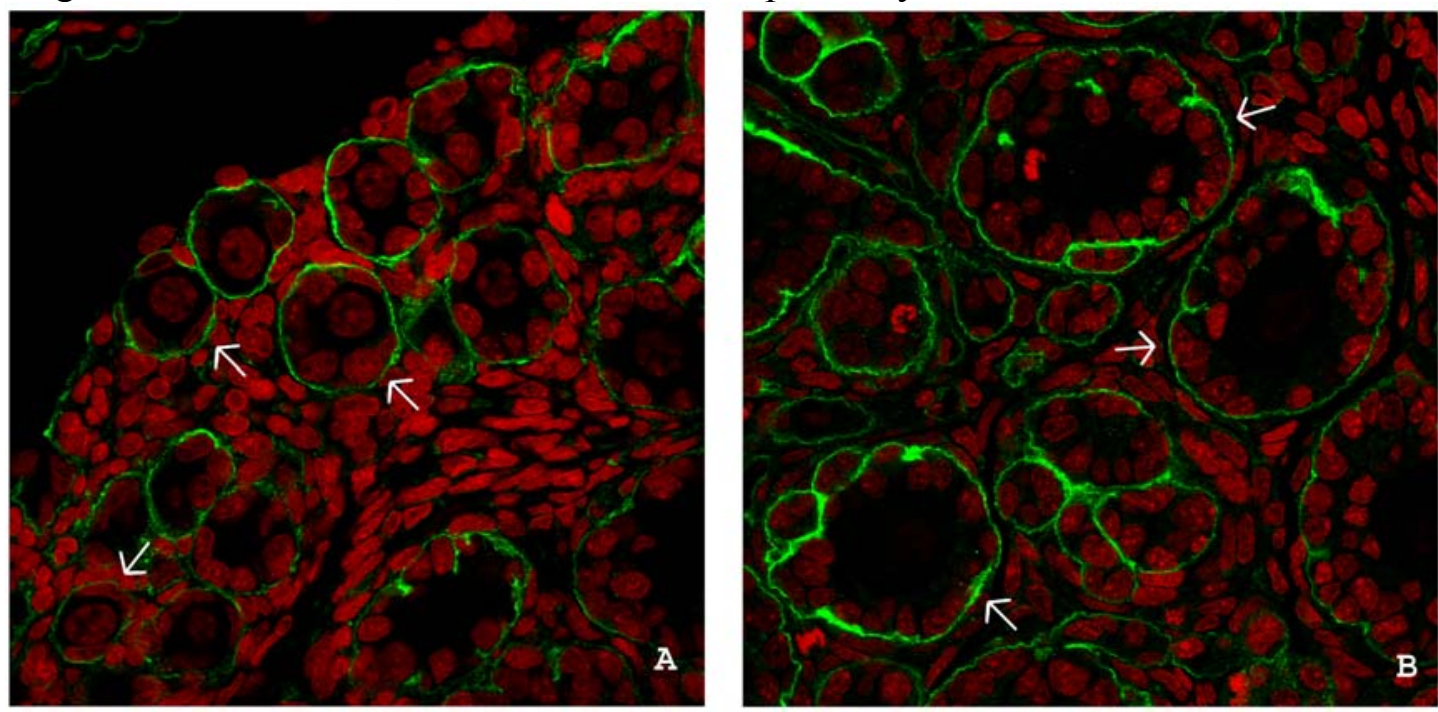

FIG 3: Immunohistochemical localization of laminin in the basement membrane of ovarian follicles from adult rats. Sections were stained with Goat anti-Laminin (Green) and Draq-5 (Red). A) Primordial follicles (arrows). B) Primary follicles (arrows). Both images were taken at a magnification of $1000 x$. 